\title{
TWO TORSION IN $H$-SPACES
}

\author{
BY JOHN R. HARPER AND JAMES P. LIN
}

Communicated by E. H. Brown, Jr., February 13, 1976

The objective of this note is to announce theorems about two torsion in $H$-spaces. We say that $X$ is a finite $H$-space if it has the homotopy type of a finite CW complex. Throughout this paper we assume that $X$ is any simply connected, finite $H$-space whose mod two homology ring $H_{*}\left(X ; Z_{2}\right)$ is associative. Every known finite simply connected $H$-space satisfies this assumption.

THEOREM 1. Let $\Omega X$ be the space of basepointed loops on $X$. Then $H_{*}(\Omega X ; Z)$ has no two torsion, and $H_{*}\left(\Omega X ; Z_{2}\right)$ is concentrated in even degrees.

THEOREM 2. The two torsion coefficients of $H^{*}(X ; Z)$ are of order at most two.

THEOREM 3. There are no even degree algebra generators in the mod two cohomology ring, $H^{*}\left(X ; Z_{2}\right)$.

Theorem 4 (Hurewicz MAP). The kernel of the two-local Hurewicz homomorphism $h_{*} \otimes Z_{(2)}: \Pi_{*}(X) \otimes Z_{(2)} \rightarrow H_{*}\left(X ; Z_{(2)}\right)$ is the two torsion of $\Pi_{*}(X)$.

Thus, the first nonvanishing homotopy group of $X$ is two torsion free.

In the case that $X$ is a simply connected Lie group, the above results were proven by appealing to the underlying differentiable manifold structure of the group. Details and proofs will appear elsewhere.

\section{REFERENCES}

1. R. Bott, On torsion in Lie groups, Proc. Nat. Acad. Sci. U.S.A. 40 (1954), 586588. MR 16, 12 .

2. J. R. Harper, Homotopy groups of H-spaces. I, Comment. Math. Helv. 47 (1972), 311-331. MR 49 \#6231.

3. J. Lin, H-spaces with finitely generated cohomology algebras, Bull. Amer. Math. Soc. 80 (1974), 1233-1238.

4. The loop space problem and its consequences, Bull. Amer. Math. Soc. $81(1975), 723-725$.

5. - Torsion in H-spaces. I, Ann. of Math. (to appear).

DEPARTMENT OF MATHEMATICS, UNIVERSITY OF ROCHESTER, ROCHESTER NEW YORK 14627

DEPARTMENT OF MATHEMATICS, UNIVERSITY OF CALIFORNIA, SAN DIEGO, LA JOLLA, CALIFORNIA 92093

AMS (MOS) subject classifications (1970). Primary 57F25, 55D45, 55G20, Secondary 57F05, 57F10, 55J20. 